\title{
Terapia con tracto vocal semi-ocluido: Un estudio de caso
}

\author{
Voice therapy with semi-occluded vocal tract: a case \\ study
}

\section{Marco Guzmán N.}

Fonoaudiólogo

Especialista en Vocología, Universidad of lowa

Académico

Escuela de Fonoaudiología Universidad de Chile
RESUMEN

Introducción: Los ejercicios de tracto vocal semi-ocluido hacen referencia a una serie de posturas que buscan alargar u ocluir el tracto vocal, generando de esta forma un cambio en el patrón vibratorio de los pliegues vocales. El mecanismo que explicaría los efectos percibidos después y durante el uso de estos ejercicios es el incremento de la inertancia del tracto vocal. Objetivo: El propósito de este trabajo es mostrar la aplicación de una secuencia de ejercicios de tracto vocal semi-ocluido como parte de un programa de tratamiento vocal y su efecto terapéutico en la fatiga vocal. Método: Estudiante de canto diagnosticado con fatiga vocal, presenta en el examen laringoscópico acumulación de mucus espeso en la glotis fonatoria y contracción laríngea antero-posterior. El plan terapéutico incluyó una secuencia de tareas fonatorias utilizando tubos de resonancia, programa de hidratación laríngea y eliminación del hábito de carraspeo. Resultados: Después de un mes se observa eliminación de la fatiga vocal, eliminación de la dificultad en los extremos de la tesitura, disminución de la mucosidad en la laringe y necesidad de aclarar la voz. Conclusiones: Los ejercicios con tracto vocal semi-ocluido pueden ser una herramienta efectiva en sujetos que presentan fatiga vocal. Estos ejercicios minimizan el esfuerzo muscular laríngeo, promoviendo una economía vocal.

Palabras claves: terapia vocal, impedancia tracto vocal, fatiga vocal

\section{ABSTRACT}

Introduction: Semi-occluded vocal tract exercises, involve a series of postures with extended and/or occluded vocal tract, producing a change in the vibration pattern of the vocal folds. The mechanism that explains the perceived effects during and after using these exercises is the increased vocal tract inertance. Objective: The purpose of this work is to show the application of a semi-occluded vocal tract exercise sequence as part of a voice treatment program and its therapeutic effect on vocal fatigue. Method: Singing student diagnosed with vocal fatigue presents mucus accumulation in phonatory glottis and antero-posterior contraction during a laringoscopy examination. The treatment plan included a phonatory sequence with resonance tubes, laryngeal hydration program and elimination of the clearing throat habit. Results: After one month of treatment, elimination of vocal fatigue, elimination of the difficulty production in the high vocal range, decreased mucus in the larynx and decreased clearing throat habit is observed. Conclusion: Semioccluded vocal tract exercises can be an effective tool in subjects with vocal fatigue. These exercises minimize laryngeal muscular effort to promote vocal economy.

Key words: voice therapy, vocal tract impedance, vocal fatigue. 


\section{Introducción}

Los ejercicios de tracto vocal semi-ocluido hacen referencia a una serie de posturas que buscan alargar u ocluir el tracto vocal, generando de esta forma un cambio en el patrón vibratorio de los pliegues vocales. Este tipo de ejercicios ha sido ampliamente utilizado por terapeutas durante toda la historia del entrenamiento y terapia vocal. Sin embargo, recién en las últimas décadas ha habido un desarrollo científico intentando explicar los principios físicos y fisiológicos detrás de los efectos atribuidos al uso de este grupo de ejercicios vocales. Algunos de los ejercicios pertenecientes al grupo de tracto vocal semi-ocluido son: humming, vibración labial, vibración lingual, fonación con consonantes fricativas labiodentales, consonantes bilabiales fricativas 0 explosivas, vocales cerradas, oclusión de la boca con la mano y uso de tubos de resonancia.

Se han realizado varios estudios para examinar los efectos de los ejercicios con tracto vocal semi-ocluido. Gaskill ${ }^{1}$ estudió las modificaciones de cociente de contacto por efecto de ejercicios de vibración labial en dos grupos, personas con y sin entrenamiento vocal. La mayoría de los sujetos mostraron una disminución del cociente de contacto de los pliegues vocales durante la realización de estos ejercicios. Miller ${ }^{2}$ estudio el efecto de las oclusiones del tracto vocal en el área de contacto de los pliegues vocales y las presiones de aire inmediatamente arriba y debajo de las glotis. El autor concluyó que algunos de los ejercicios utilizados produjeron un aumento de la amplitud de la señal electroglotográfica y un incremento del área de contacto de los pliegues vocales. Esto implicaría que el incremento de la reactancia del tracto vocal, provocada por el uso de posturas semi-oclusivas asiste la vibración y aumenta la amplitud vibratoria de las cuerdas vocales ${ }^{3}$. Utilizando dos ejercicios con tracto vocal semiocluido (Finger kazoo y fonación en tubos), Sampaio ${ }^{4}$ demostró los efectos inmediatos de ambos. Se obtuvieron resultados positivos y similares en la valoración acústica y autovaloración perceptual. En la evaluación acústica se encontró un descenso de la frecuencia fundamental y la evaluación perceptivoauditiva indicó mejorías perceptibles después de la fonación en tubos de resonancia.

La fonación dentro de tubos de resonancia ha sido usada en la terapia vocal en Finlandia desde la década de los sesenta. Este tipo de ejercicios han sido utilizados también por personas con voces normales en entrenamiento y calentamiento de la $v o z^{5}$. En este último grupo se ha observado que cantantes logran una emisión más clara, brillante y resonante después del uso de tubos de resonancia ${ }^{6}$. Un beneficio ofrecido por los tubos de resonancia de diámetro estrecho, que otros ejercicios como la vibración labial o lingual no ofrecen, es que se puede monitorear el sonido de la laringe de manera fácil, por el hecho de no ser enmascarado por el sonido de las vibraciones de labios o lengua ${ }^{7}$. En el ámbito terapéutico, los tubos de resonancia han sido aplicados con resultado positivo en casos de disfonía funcional (hiper e hipofuncional), en paresia unilateral de nervio laríngeo recurrente, en pacientes con nódulos, entre otras patologías vocales ${ }^{8}$. 
El mecanismo que explicaría los efectos percibidos después y durante el uso de ejercicios con tracto vocal semi-ocluido sería el incremento de la impedancia del tracto vocal producida por el aumento de su carga acústica. Titze ${ }^{9}$ reportó a través de una simulación computarizada que la fonación puede ser producida en forma más eficiente $y$ económica a través de la interacción entre la fuente de voz (pliegues vocales) y el filtro (tracto vocal), por el uso de técnicas terapéuticas que involucran semioclusión de los labios o una combinación de ajustes en la aducción de los pliegues vocales y del tubo epilaríngeo.

El propósito de este trabajo es mostrar la aplicación de una secuencia de ejercicios de tracto vocal semi-ocluido como parte de un programa de tratamiento vocal y su efecto terapéutico en el caso de un sujeto diagnosticado con fatiga vocal.

\section{Reporte del caso}

Estudiante de canto clásico de 24 años, sexo masculino, con clasificación vocal de tenor lírico. Se encuentra cursando el cuarto año de canto en la universidad. Su desarrollo vocal en voz cantada ha seguido un curso normal en todos sus parámetros según reportó el profesor de canto. Su historia clínica es positiva por alergia estacional esporádica y reflujo faringolaríngeo tratado y solucionado hace dos años (asintomático al momento de la evaluación). La historia médica no reporta hospitalizaciones ni enfermedades crónicas. El paciente no toma medicamentos, nunca ha fumado y bebe alcohol en forma ocasional. La ingesta de líquido no es adecuada. Señala beber sólo un vaso de agua al día y una taza de té cada mañana.

El paciente consulta por presentar fatiga vocal, acumulación de mucus en la laringe, necesidad de carraspear constantemente y miedo a tener algún problema en su voz. Señala que siente su voz levemente soplada, especialmente la zona media y baja de su tesitura. Las frecuencias agudas en voz de cabeza (voz cubierta o full head) son más forzadas y necesitan más presión de aire para ser emitidas. El problema se inició dos meses antes de la fecha de evaluación, según refiere el paciente.

Paralelamente a las clases regulares de canto dos veces por semana, se encuentra realizando un taller de ópera, donde canta uno de los roles principales. Este taller involucra 5 horas de ensayo semanal. 2,5 horas el día martes en la tarde y 2,5 horas en día viernes en la mañana.

Los resultados de la videoestroboscopía laríngea muestran una laringe anatómicamente normal y parámetros dinámicos dentro de rangos de normalidad. Solamente se observó acumulación de mucus espeso en la zona media de la glotis fonatoria. En la valoración laringoscópica con fibra flexible se observa leve constricción aritenoepiglótica al emitir notas en la zona aguda de la tesitura en registro de cabeza (voz cubierta o full head). En la evaluación de las medidas aerodinámicas de la fonación se obtuvo un promedio de velocidad de flujo glótico de 231 $\mathrm{ml} / \mathrm{seg}$. (rango normal 80-200 ml/seg.), presión subglótica promedio de $13 \mathrm{~cm}$. de $\mathrm{H}^{2} \mathrm{O}$ (rango normal 5-10 cm. de $\left.\mathrm{H}^{2} \mathrm{O}\right)$. Estas medidas fueron tomadas pidiéndole al sujeto una emisión en frecuencia 
fundamental e intensidad cómodas. Tanto el promedio de velocidad de flujo, como la presión subglótica están levemente sobre el rango de normalidad. La valoración de las medidas aerodinámicas se realizó con el equipo Aerodynamic Phonatory System, modelo 6600 (Kaypentax).

La evaluación funcional de la voz hablada no mostró anormalidades. Los índices acústicos jitter, shimmer, soft phonation index (SPI) y noise to harmonic ratio (NHR) obtuvieron valores normales durante la emisión de la vocal /a/ mantenida en voz hablada. En la valoración vocal de voz cantada, perceptualmente el paciente presenta un leve grado de voz con esfuerzo en la emisión en extremo superior de la tesitura (últimos tres tonos), hecho que podría estar relacionado con lo observado en la laringoscopía flexible. La tesitura del sujeto se encuentra en el rango de La 1-Do 4 (normal para su clasificación vocal). El pasaje vocal de registro de pecho a cabeza (voz cubierta) está alrededor de Fa \# 3 (normal para su clasificación vocal), no presentando dificultades técnicas. Los parámetros respiratorios no presentan alteraciones durante la emisión de voz cantada. Se destaca el buen manejo del apoyo respiratorio y fiato durante la producción de diferentes vocalizaciones de dificultad técnica variable. Perceptualmente su voz presenta una sonoridad media y brillo adecuado en la zona alta de su tesitura, sin embargo existe un leve escape de aire en la zona media y baja de la tesitura, lo que desaparece con el aumento de la intensidad. Los parámetros acústicos del vibrato se encuentran dentro de rangos normales.
El diagnóstico final del paciente en estudio fue fatiga vocal asociada a un posible cuadro de sobre carga vocal y deshidratación laríngea.

\section{Plan de tratamiento}

El tratamiento incluyó los siguientes aspectos:

1) Programa de ejercicios fonatorios con tracto vocal semi-ocluido (tubos de resonancia)

2) Programa de hidratación laríngea

3) Eliminación del aclaramiento de garganta

La secuencia de ejercicios fonatorios con tracto vocal semi-ocluido estuvo compuesta por 4 tareas fonatorias dentro de tubos de resonancia (bombillas de plástico para revolver) de $2.8 \mathrm{~mm}$. de diámetro interno y $13.5 \mathrm{~cm}$ de longitud. La figura 1 muestra la posición de la bombilla durante los ejercicios fonatorios.

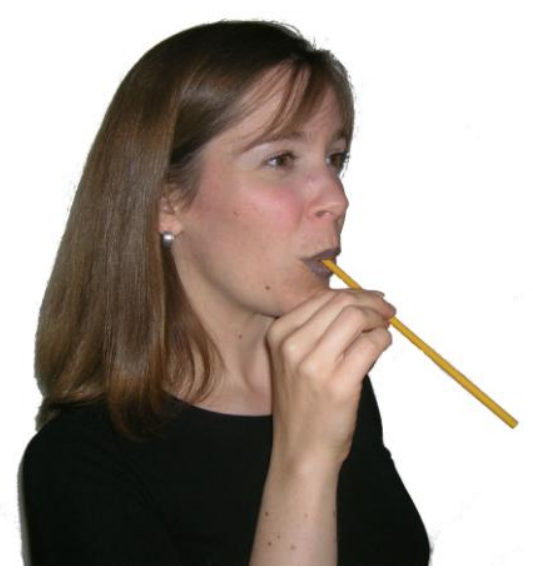

Figura 1. Posición adecuada del tubo de resonancia durante las tareas fonatorias aplicadas. 
El objetivo de esta secuencia de ejercicios es lograr un balance fisiológico de los tres subsistemas involucrados en la producción de la voz (respiración, fonación y resonancia), no centrándose en el trabajo directo sobre uno de estos tres componentes ${ }^{10}$. Este objetivo está basado en la orientación fisiológica de terapia vocal.

Las tareas fonatorias fueron las siguientes:

1) Fonación en tubo produciendo una frecuencia mantenida y cómoda para cada sujeto en intensidad y altura (Figura 2).

2) Fonación en tubo produciendo glissandos ascendentes y descendentes (Figura 3).

3) Fonación en tubo haciendo acentos de intensidad y frecuencia con utilización de la musculatura abdominal. La realización de los acentos fue en forma ascendente (Figura 4).

4) Producción de la melodía de una canción a la elección del sujeto dentro del tubo (Figura 5).

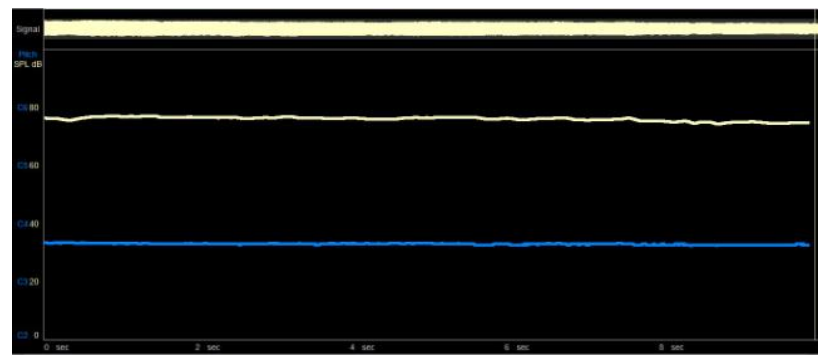

Figura 2. Diagrama de fonación en tubo produciendo una frecuencia mantenida y cómoda para cada sujeto en intensidad y altura. La línea inferior señala la frecuencia fundamental y la superior la intensidad.

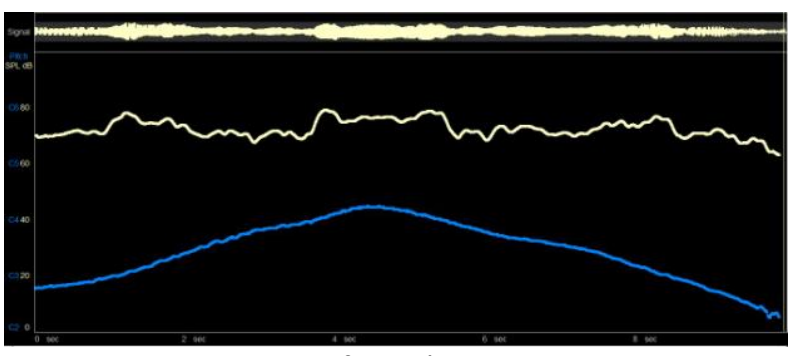

Figura 3: Diagrama de fonación en tubo produciendo glissandos ascendentes y descendentes. La línea inferior señala la frecuencia fundamental y la superior la intensidad.

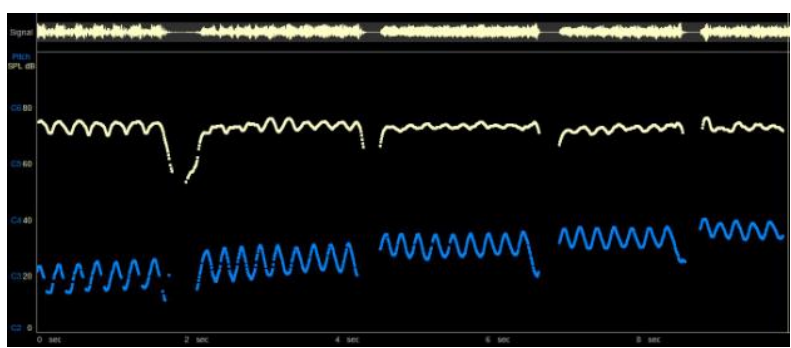

Figura 4: Diagrama de fonación en tubo haciendo acentos de intensidad y frecuencia con utilización de la musculatura abdominal. Realización de los acentos en forma ascendente. La línea inferior señala la frecuencia fundamental y la superior la intensidad.

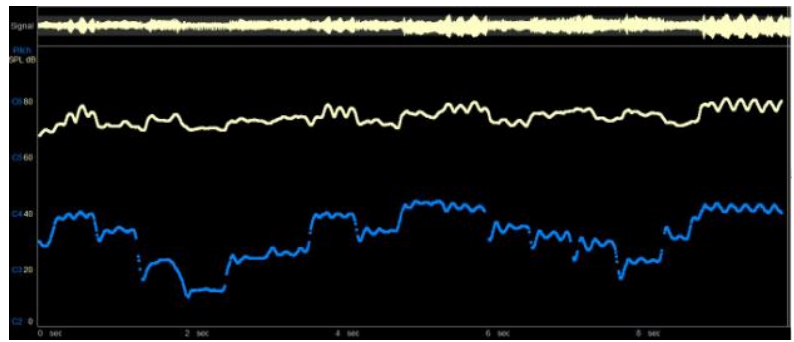

Figura 5: Diagrama de la producción de la melodía de una canción dentro del tubo. La línea inferior señala la frecuencia fundamental y la superior la intensidad.

Se solicitó al sujeto mantener la sensación de "garganta abierta" durante la realización de los ejercicios. Esto implica descenso laríngeo, protrusión labial, descenso mandibular y descenso lingual, lo que produce un mayor alargamiento del tracto vocal (similar a la voz cubierta). 
La duración de la secuencia completa de ejercicios fue de 12 minutos. Cada tarea fonatoria se realizó durante 3 minutos. Se permitió que el paciente pudiera inspirar las veces que fuera necesario durante la realización de cada ejercicio. Se realizaron 4 sesiones de terapia vocal (durante un mes) con una duración de 30 minutos cada una. La frecuencia fue de una sesión semanal, más indicaciones de realización de los ejercicios 3 veces al día en el hogar. Además se indicó realizar los ejercicios una vez antes y después de cantar a modo de calentamiento y enfriamiento vocal respectivamente.

El programa de hidratación laríngea fue el siguiente: beber a lo menos dos litros de agua al día de forma fragmentada. La temperatura del agua debe ser temperatura ambiente. Se indicó que esta cantidad debía ser aumentada durante las horas previas a cantar, en un régimen de cuatro vasos de agua durante las dos horas previas a los ensayos o clases de canto. El objetivo de este aumento en la ingesta de agua es favorecer una mucosa laríngea en mejor condición vibratoria para el calentamiento vocal y la actuación. La realización de vaporizaciones e ingesta de medicamentos mucolíticos son recomendables dentro de un buen programa de hidratación laríngea. Sin embargo, es este caso particular sólo se utilizó la hidratación sistémica a través de la ingesta de agua.

La eliminación del hábito de aclarar la garganta se realizó solicitando al sujeto que en lugar de carraspear, tomara un trago de agua produciendo una deglución exagerada y con fuerza. Se indicó que durante esta maniobra, la cabeza y cuello deben estar inclinados hacia adelante, de tal forma que el mentón toque en pecho. Si la sensación de mucosidad continuaba, se debía realizar una tos áfona con el objetivo de remover la mucosidad de los pliegues vocales.

\section{Resultados}

Después de 4 sesiones de trabajo, más la práctica diaria en casa, los resultados mostraron la eliminación de la voz soplada (según información entregada por el paciente y percepción del clínico), eliminación de la fatiga vocal y eliminación de la dificultad fonatoria en los extremos de la tesitura. Además, el sujeto señaló una disminución considerable de la mucosidad en la laringe y disminución de la necesidad de aclarar la voz.

Los resultados de la videoestroboscopía laríngea evidenciaron una disminución de la acumulación de mucus en los pliegues vocales. En la valoración laringoscópica con fibra flexible se mantuvo la leve constricción aritenoepiglótica al emitir notas en la zona aguda de la tesitura en registro de cabeza.

En la evaluación de las medidas aerodinámicas de la fonación se obtuvo un promedio de velocidad de flujo glótico de $107 \mathrm{ml} / \mathrm{seg}$. (Rango normal 80-200 $\mathrm{ml} / \mathrm{seg}$.) y presión subglótica promedio de $8 \mathrm{~cm}$. de $\mathrm{H}^{2} \mathrm{O}$ (Rango normal 5-10 cm. de $\mathrm{H}^{2} \mathrm{O}$ ).

El paciente fue dado de alta con sugerencia de control en 6 meses más o antes si los síntomas se manifiestan nuevamente. 


\section{Discusión}

El presente trabajo presenta el caso de un estudiante de canto clásico con problemas fonatorios en voz cantada asociados a un cuadro de fatiga vocal, posiblemente debido a sobrecarga vocal. Considerando que la fatiga vocal puede ser un estadio temprano de una alteración vocal más estructurada, es importante abordarla tempranamente. En este caso el tratamiento comenzó dos meses después de la aparición de los primeros síntomas.

De acuerdo a Titze ${ }^{11}$, la fatiga vocal puede mostrar signos tales como aumento del mucus laríngeo, necesidad excesiva de aclarar la voz (carraspear), ronquera, voz soplada, disminución de la intensidad e inestabilidad de la voz. La sintomatología del paciente en estudio coincide en mayoría de los aspectos señalados por Titze. En la evaluación inicial el paciente manifestó la necesidad de aclarar la garganta en forma frecuente y exceso de mucus, hecho que se corroboró mediante la evaluación videoestroboscópica, donde se observó mucus espeso. Después de un mes de tratamiento, esta sintomatología disminuyó en forma considerable subjetiva y objetivamente. Es posible atribuir este cambio tanto al programa de hidratación laríngea como a la práctica de ejercicios con tracto vocal semiocluido. Titze $^{11}$ señala que la fatiga vocal puede manifestarse en cantantes con pérdida o dificultad en las frecuencias agudas de la tesitura, signo que también estuvo presente en el sujeto estudiado. Finalizado el tratamiento este problema técnico desapareció, probablemente como consecuencia de la eliminación de la fatiga vocal. En un estudio de caso con un paciente con fatiga vocal, Stemple ${ }^{12}$ utilizó estrategias similares a las utilizadas en el presente trabajo. El autor realizó tratamiento de hidratación laríngea sistémica, eliminación del hábito de aclarar la garganta, higiene vocal y además aplicó un programa de ejercicios llamado Vocal Function Exercises, el cual está basado en tareas fonatorias con tracto vocal semi-ocluido. Los resultados mostraron que a través del plan terapéutico aplicado se logró eliminar el cuadro de fatiga vocal.

Si bien el plan de tratamiento en el presente reporte estuvo compuesto de tres aspectos, el elemento central fue la secuencia de ejercicios fonatorios con tracto vocal semi-ocluido. La primera tarea fonatoria, mantención de un tono cómodo, fue utilizada como una actividad de calentamiento vocal, previo a las tres siguientes de mayor complejidad y exigencia muscular vocal. Con la segunda tarea fonatoria, realización de glissandos ascendentes y descendentes, se busca respectivamente la elongación y la contracción de los pliegues vocales. Estos dos últimos ejercicios también son parte importante de otros programas de ejercicios vocales desarrollados con anterioridad ${ }^{10}$. La tercera tarea fonatoria de la secuencia utilizada fue la realización de acentos de intensidad y frecuencia, logrando a través de ellos nuevamente una elongación de los pliegues vocales en forma progresiva y paralelamente cambios de presión subglótica a través de impulsos abdominales que debía producir el paciente durante su realización.

Finalmente, la fonación de la melodía de una canción fue utilizada como una actividad que tiene 
como objetivo unir las tres anteriores. Al producir una melodía se mantiene la fonación, se producen elongaciones y contracciones de los pliegues vocales en forma constante $\mathrm{y}$ en todo momento ocurren variaciones de presión subglótica por los cambios de intensidad y frecuencia.

Todos los ejercicios fueron realizados dentro de un tubo (bombilla), utilizado como una extensión artificial del tracto vocal, aumentando de esta forma la carga acústica $^{13}$ y obteniendo los efectos fisiológicos y acústicos descritos en la literatura. Las razones de porqué los ejercicios con tracto vocal semi-ocluido (alargamientos) pueden haber sido útiles en este caso de fatiga vocal, son variadas. Behlau $^{14}$ afirma que una oclusión parcial en la boca promueve una resonancia retro refleja y expansión de toda el área del tracto vocal, la boca y la laringe. Algunos estudios han propuesto que la carga del tracto vocal o un aumento de la reactancia inertiva, $^{15,16}$ pueden afectar la vibración de los pliegues vocales de una manera favorable, ${ }^{15,17}$ lográndose un balance muscular. Titze ${ }^{9}$ reportó a través de una simulación computarizada que la fonación puede ser producida en forma más eficiente y económica a través de la interacción entre la fuente de voz (pliegues vocales) y el filtro (tracto vocal), por el uso de técnicas terapéuticas que involucran semioclusión de los labios.

El objetivo terapéutico del caso presentado fue eliminar el cuadro de fatiga vocal a través de un programa centrado en ejercicios de tracto vocal semiocluido. La secuencia utilizada probablemente produjo una diminución del trabajo muscular necesario para la fonación, puesto que estos ejercicios favorecen una fonación más eficiente $y$ económica, lo que implica en términos prácticos, mayor exteriorización del sonido, con menos estrés mecánico de los pliegues vocales producido por la colisión entre ellos. De esta forma el sujeto logró una producción de voz sin involucrar esfuerzo muscular compensatorio.

Varios estudios han sido realizados para conocer los efectos fisiológicos de los ejercicios con tracto vocal semi-ocluido en relación a la disminución del esfuerzo fonatorio. Gaskill ${ }^{1}$ estudió las modificaciones de cociente de contacto por efecto de ejercicios de vibración labial en dos grupos, personas con y sin entrenamiento vocal. La mayoría de los sujetos mostraron una disminución del cociente de contacto de los pliegues vocales durante la realización de estos ejercicios. El efecto fue mayor en el grupo de sujetos sin entrenamiento vocal previo. En un estudio reciente, Gaskill ${ }^{13}$ analizó el efecto del alargamiento artificial del tracto vocal en el cociente de contacto glótico estimado en voces no entrenadas. Los resultados mostraron cambios significativos en el cociente de contacto durante la fonación en tubos, pero sin un patrón claro a través de los 15 sujetos participantes. Los resultados de estos estudios podrían constituir un hecho importante en cuadros de fatiga vocal. El aumento del cociente de contacto de los pliegues vocales puede formar parte del grupo de causas de la fatiga, así como también, puede ser un mecanismo compensatorio (consecuencia) cuando el cuatro de fatiga vocal ya está instaurado. En ambos casos, el uso de semi-oclusiones del tracto vocal sería una herramienta terapéutica deseada con el propósito de 
provocar una disminución del esfuerzo fonatorio en sujetos que presentan fatiga vocal o cualquier cuadro vocal patológico que involucre un aumento del grado de aducción de los pliegues vocales. En el presente estudio no se midió el cociente de contacto, sin embargo, es posible que éste haya experimentado cambios considerando los reportes recién señalados.

Una posible explicación fisiológica de la disminución del cociente de contacto durante el uso de ejercicios con semi-oclusión es entregada por Titze $^{7}$. El autor investigó el efecto de los tubos de resonancia en el calentamiento vocal en cantantes, concluyendo que con el uso de los tubos de resonancia disminuyen las fuerzas de colisión de los pliegues vocales y la fonación apretada no es posible durante el ejercicio. Por el contrario, estos ejercicios separan levemente los pliegues vocales. Uno de los efectos aerodinámicos producidos por el uso de tubos o estrechamientos del tracto vocal es el incremento del promedio de la presión supraglótica y por lo tanto un aumento de la presión intraglotal. Esto tiende a separar los pliegues vocales, reduciendo el impacto mecánico al contactarse medialmente ${ }^{7}$.

$$
\text { Complementando lo anterior, Titze }{ }^{18} \text { señala }
$$
que con el uso de ejercicios de tracto vocal semiocluido se produce un descenso del umbral de presión de fonación (presión subglótica mínima necesaria para iniciar o mantener la fonación), lo que causa una producción vocal más económica. La disminución del umbral de presión de la fonación se refleja de manera práctica en la disminución del esfuerzo durante el proceso de producción de la voz. En el paciente analizado en el presente estudio, no se midió el umbral de presión, sin embargo, fue evaluada una medida relacionada, la presión subglótica. El sujeto mostró una disminución de la presión subglótica de 13 a $8 \mathrm{~cm}$. de $\mathrm{H}^{2} \mathrm{O}$ (rango normal 5-10 cm. de $\left.\mathrm{H}^{2} \mathrm{O}\right)$. Esta disminución también podría estar asociada a una disminución del esfuerzo fonatorio, considerando que es común observar un aumento de esta presión en cuadros de fatiga vocal como mecanismo compensatorio fisiológico.

Relacionado también con la disminución del esfuerzo fonatorio, Laukkanen ${ }^{19}$ investigó el efecto de la consonante fricativa bilabial / $/$ :. Los resultados mostraron descenso laríngeo, actividad muscular laríngea reducida, pendiente más acentuada en el espectro de la voz y mayor economía vocal.

De acuerdo a la evidencia científica señalada, se puede deducir que el uso de los tubos de resonancia en el presente estudio favoreció una fonación más fácil y relajada, producida probablemente por la disminución del cociente de contacto, descenso del umbral de presión, disminución de la colisión de los pliegues vocales y, en general, una reducción de la actividad muscular laríngea. Como consecuencia del balance muscular laríngeo logrado, se obtuvieron cambios aerodinámicos fonatorios objetivos tales como la disminución del promedio de flujo glótico (107 $\mathrm{ml} / \mathrm{seg}$ ) y disminución de la presión subglótica $(8 \mathrm{~cm}$. $\mathrm{H}_{2} \mathrm{O}$ ), estando ambos parámetros dentro de rangos de normalidad al finalizar el tratamiento. Es posible que el aumento de la presión subglótica y flujo fonatorio detectados en la evaluación inicial hayan sido el reflejo un comportamiento compensatorio muscular frente a la diminución de la resistencia laríngea causada por el cuadro de fatiga vocal. A 
través de los ejercicios con tracto vocal semi-ocluido se logró una disminución del esfuerzo fonatorio y a partir de esa mejoría, un balance de los tres subsistemas involucrados en la producción de la voz (fonación, respiración y resonancia).

En la valoración laringoscópica con fibra flexible se mantuvo la leve constricción aritenoepiglótica al emitir notas en la zona aguda de la tesitura en registro de cabeza (full head). Existe la posibilidad que este patrón de contracción supraglótica no haya sido parte de la sintomatología del cuatro de fatiga vocal. Estudios señalan que este tipo de comportamiento laríngeo forma parte normal de la fisiología de las voces con entrenamiento vocal clásico y son un aspecto importante en la producción de las voces resonantes y brillantes. ${ }^{20,21}$

Parte complementaria importante del plan de tratamiento en este estudio fue la hidratación laríngea. El aumento de viscosidad y cantidad del mucus laríngeo, además de ser un síntoma de fatiga vocal, es una señal de falta de hidratación ${ }^{14}$. Existen varias razones fisiológicas que soportan la idea que la hidratación de los pliegues vocales debe ser parte del tratamiento vocal. Los pliegues vocales están cubiertos por una delgada capa de líquido ${ }^{22}$. Este líquido sirve como una barrera física y biomecánica que protege los tejidos subyacentes de partículas y patógenos inhalados ${ }^{23}$. La presencia de líquido en la superficie es útil además para mantener las características biomecánicas de la mucosa de los pliegues vocales en forma óptima, incrementar la eficiencia de la oscilación y promover una cualidad de voz normal ${ }^{24,25}$. Behlau ${ }^{14}$ señala que para mejorar el grado de hidratación laríngea es indicado ingerir entre 2.5 a 3.0 litros de agua al día. Además sugiere que en los profesionales de la voz esta cantidad debe ser aumentada antes de la actuación. Similar a lo planteado por este autor, el programa aplicado en este reporte consideró una ingesta base de agua al día y además un aumento de esta cantidad durante las horas previas a las actividades de voz cantada. Es probable que el programa de hidratación aplicado también haya contribuido a la producción de una fonación más eficiente, vibración de los pliegues vocales adecuada, y por lo tanto, reducción del esfuerzo muscular.

\section{Conclusión}

Los ejercicios con tracto vocal semi-ocluido, particularmente el uso de tubos de resonancia como parte del plan de tratamiento, pueden ser efectivos en sujetos que presentan fatiga vocal. Estos ejercicios minimizan el esfuerzo muscular laríngeo, promoviendo una economía vocal.

\section{Referencias}

1. Gaskill, C. Erickson, M. 2008. The effect of a voiced lip trill on estimated glottal closed quotient. J Voice, 22, 634-43.

2. Miller, D. Schutte, H. 1991. Effects of downstream occlusions on pressures near the glottis in singing. En Gauffin, J. Hammarberg, X. Vocal fold physiology. Acoustic, perceptual and physiological aspects of voice mechanism. Stockholm, Royal Institute of Technology: Singular Publishing Group.

3. Peterson, K. Verdolini, K. Barkmeier, J. Hoffman, H. 1994. Comparison of aerodynamic and electroglottographic parameters in evaluating clinically 
relevant voicing patterns. Ann Oto Rhin Lar, 103, 33546.

4. Sampaio, M. Oliveira, G. Behlau, M. 2008. Investigation of immediate effects of two semi-ocluded vocal tract exercises. Pro-Fono, revista de actualización cientifica, 20, 261-266.

5. Simberg, S. Laine, A. 2007. The resonance tube method in voice therapy: Description and practical Implementations. Logop Phon Vocology, 32, 165-170.

6. Laukkanen, A. Titze, I. Hoffman, H. Finnegan, E. 2008. Effects of a Semioccluded Vocal Tract on Laryngeal Muscle Activity and Glottal Adduction in a Single Female Subject. Folia Phoniatr Logop, 60, 298-311.

7. Titze, I. Finnegan, E. Laukkanen, A. Jaiswal, S. 2002. Raising lung pressure and pitch in vocal warm-ups: the use of flow-resistant straws. J Singing, 58, 329-338.

8. Simberg, S. 2004. Prevalence of vocal symptoms and voice disorders among teacher students and teachers and a model of early intervention. Finland: University of Helsinki, Department of Speech Sciences.

9. Titze, I. 2006. Voice training and therapy with a semioccluded vocal tract: rationale and scientific underpinnings. J Speech Lang Hear Res, 49, 448-459.

10. Stemple, J. Glaze, L. Klaben, B. 2010. Clinical voice pathology, theory and management. Canada: Singular Thomson Learning.

11. Titze, I. 1994. Principles of Voice Production. Englewood Cliffs, NJ: Prentice Hall.

12. Stemple, J. 2000. Voice therapy clinical studies. Canada: Singular Thomson learning.

13. Gaskill, C. Erickson, M. 2010. The effect of an artificially lengthened vocal tract on estimated glottal contact quotient in untrained male voices. J Voice, 24, 57-71.

14. Behlau, M. 2005. Voz, o libro do especialista, Vol II. Rio de Janeiro: Revinter.

15. Titze, I. 1988. The physics of small-amplitude oscillation of the vocal folds. J Acoust Soc Am, 83, 1536-1552.

16. Story, B. Laukkanen, A. Titze, I. 2000. Acoustic impedance of an artificially lengthened and constricted vocal tract. J Voice, 14, 455-469.
17. Titze, I. Story, B. 1997. Acoustic interactions of the voice source with the lower vocal tract. J Acoust Soc Am, 101, 2234-2243.

18. Titze, I. 2008. Nonlinear source-filter coupling in phonation theory. J Acoust Soc Am, 123, 2733-2749.

19. Laukkanen, A. Lindholm, P. Vilkman, E. et al. 1996. A physiological and acoustic study on voiced bilabial fricative /ß:/ as a vocal exercise. J Voice, 10, 67-77.

20. Pershall, K. Boone, D. 1987. Supraglottic contribution to voice quality. J Voice, 1, 186-190.

21. Yanagisawa, E. Estill, J. Kmucha, S. Leder, S. 1989. The contribution of aryepiglotic constriction to "ringing" voice quality-A videolaryngoscopic study with acoustic analysis. $J$ Voice, 3, 242-350.

22. Fukuda, H. Kawaida, M. Tatchara, T. Ling, E. Kita, K. Ohki, K et al. 1988. A new concept of lubricating mechanisms of the larynx. En Fujimara, O. Vocal Fold Physiology: Voice Production, Mechanisms, and Functions. New York, NY: Raven Press Ltd.

23. Mogi, G. Watanabe, N. Maeda, S. Umehara, T. 2002. Laryngeal secretions. An immunochemical and immunohistological study. Otolaryngol Head Neck Surg, 126, 528-537.

24. Hemler, R. Wienke, G. Dejonckere, P. 1997. The effect of relative humidity of inhaled air on acoustic parameters of voice in normal subjects. J Voice, 11, 295-300.

25. Chan, R. Tayama, N. 2002. Biomechanical effects of hydration in vocal fold tissues. Otolaryngol Head Neck Surg, 126, 528-537. 